\title{
Single Nanowire Terahertz Detectors
}

\author{
Kun Peng ${ }^{1}$, Patrick Parkinson ${ }^{2}$, Lan Fu ${ }^{1}$, Qiang Gao ${ }^{1}$, Nian Jiang ${ }^{1}$, Ya-Nan Guo ${ }^{1}$, Fan Wang ${ }^{1}$, Hannah J \\ Joyce $^{2,3}$, Jessica L Boland ${ }^{2}$, Michael B Johnston ${ }^{2}$, Hark Hoe Tan ${ }^{1}$, Chennupati Jagadish ${ }^{1}$ \\ 1.Department of Electronic Materials Engineering, Research School of Physics and Engineering, \\ The Australian National University, Canberra, ACT 0200, Australia \\ 2.Department of Physics, University of Oxford, Clarendon Laboratory, Oxford OX1 3PU, UK \\ 3.Department of Engineering, University of Cambridge, $9 \mathrm{JJ}$ Thomson Avenue, Cambridge CB3 OFA, UK \\ Corresponding author: Kun Peng, phone: +612 61251593; Fax: +612 61250511; e-mail: kun.peng@anu.edu.au
}

\begin{abstract}
Photoconductive terahertz detectors based on single GaAs/AlGaAs nanowire were designed, fabricated and incorporated into the pulsed time-domain technique, showing a promise for nanowires in terahertz applications such as near-field terahertz sensors or on-chip terahertz micro-spectrometers.

OCIS codes: (040.5160) Photodetectors; (160.4236) Nanomaterials; (300.6495) Spectroscopy, terahertz
\end{abstract}

\section{Introduction}

Terahertz (THz) spectroscopy and imaging have been widely used for study of structure and dynamics in diverse materials, security screening, biology imaging and non-destructive testing [1, 2]. However, the lack of traditional materials for $\mathrm{THz}$ generation and detection has limited the development of this field. Photoconductive (Auston) switches technique [3] based on semiconductor materials by using ion implantation-induced damaged or low temperature grown semiconductor materials, is commonly used for $\mathrm{THz}$ detection in current $\mathrm{THz}$ system. Onedimensional III-V compound semiconductor nanowires, due to the nano-scale size, direct and tunable band gap, high carrier mobility (close to that in bulk materials), and in contrast short carrier lifetime (typically sub-nanoseconds) [2,4] leading to a fast response time in the $\mathrm{THz}$ regime, have been considered as an ideal alternative to bulk semiconductor materials for $\mathrm{THz}$ detection, particularly to be used as elements for highly integrated nanoscale $\mathrm{THz}$ devices such as a sub-wavelength detector element for near-field imaging or integrated into an "on-chip" THz spectrometer [5]. In this work, we demonstrate the design, fabrication and characterization of single GaAs/AlGaAs nanowire photoconductive $\mathrm{THz}$ detectors.

\section{Nanowire growth and detector fabrication}

The GaAs/AlGaAs core-shell nanowires were grown with metalorganic chemical vapour deposition (MOCVD) technique via the vapour-liquid-solid (VLS) mechanism [4,6]. Fig. 1a demonstrates a schematic cross-section diagram of a typical single GaAs/AlGaAs core-shell nanowire used in our detectors and corresponding TEM image. Fig. $1 \mathrm{~b}$ is a SEM image of the nanowire. Photoluminescence (PL) measurements were performed to characterize the optical properties of such nanowires (see Fig1c). To fabricate the single-nanowire detectors, the as-grown substrate was cleaved into small pieces and placed in isopropyl alcohol solution for a 30-second ultra-sonication to transfer the nanowires into solution. The solution was dropped onto z-cut quartz substrates and allowed to dry naturally. Then the quartz substrates were spin-coated with photoresist and patterned using direct-laser-write lithography technique [7]. At last, the detector structure was metallized using electron beam evaporation to form 10/300nm $\mathrm{Ti} / \mathrm{Au}$ contacts on each side of the nanowire.
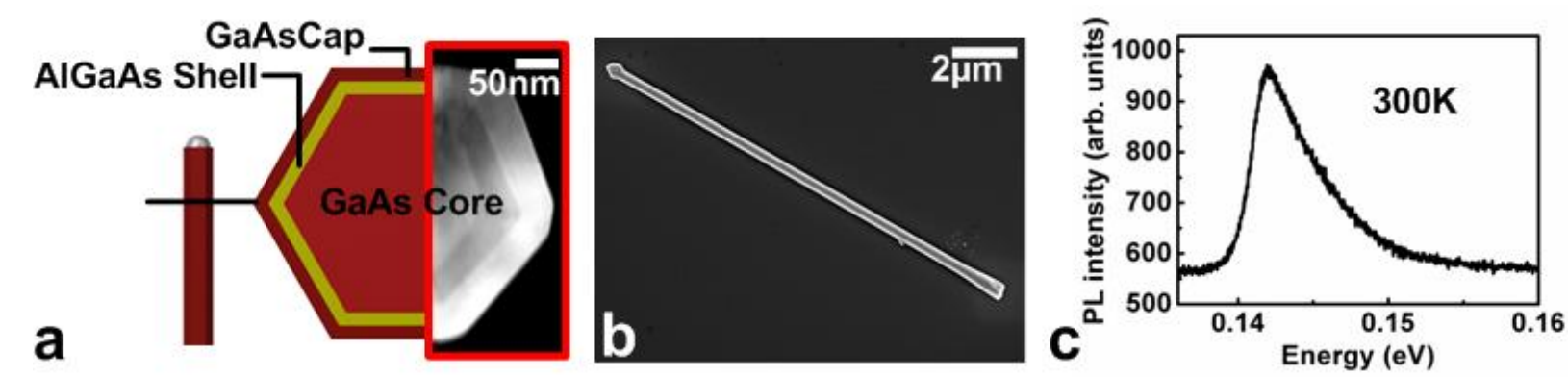

Fig. 1.a) A schematic cross-section of the GaAs/AlGaAs core-shell nanowire used in our detectors and corresponding tunneling electron micrograph image. b) Scanning electron micrograph images of the nanowire. c) A typical PL spectrum of a single core-shell nanowire.

\section{THz response characterization and finite-difference time-domain (FDTD) simulations}


The single-nanowire detectors were incorporated into a THz time-domain spectroscopy (THz-TDS) system [2] as a sensor to measure the incident $\mathrm{THz}$ pulse signal. Fig. 2a shows a schematic diagram of our single-nanowire photoconductive detector in operation. The $\mathrm{THz}$ induced photocurrents measured from our single-nanowire detectors were shown in Fig. 2b, and then processed and converted to THz spectral responses (see Fig. 2c). It is can be seen from Fig. 2c that our nanowire detector has a bandwidth in the range of 0.1 to $0.6 \mathrm{THz}$. Nevertheless, our single nanowire detector has sufficient signal-to-noise ratio for real-world application. We inserted a $290 \mathrm{GHz}$ lowpass filter into the THz-TDS system. The spectral response signal was abruptly cut off at the frequency of $290 \mathrm{GHz}$ with transmission amplitude around $80 \%$. Comparing with a standard ion-implanted InP receiver, we confirmed that our nanowire detector have good enough sensitivity for practical use and its nanoscale size will bring much more advantages for future applications. FDTD simulations [8] with the same detector geometry used in the experiments were performed in the $\mathrm{THz}$ regime to understand the origin of the narrow bandwidth of such detectors. The results from these simulations indicate that the narrowed detection bandwidth arises from the specific detector antenna design, rather than the nanowire itself. Other antenna designs such as bow-tie or strip-line structure, were also simulated, presenting the importance of a properly design of detector geometry for $\mathrm{THz}$ detection.
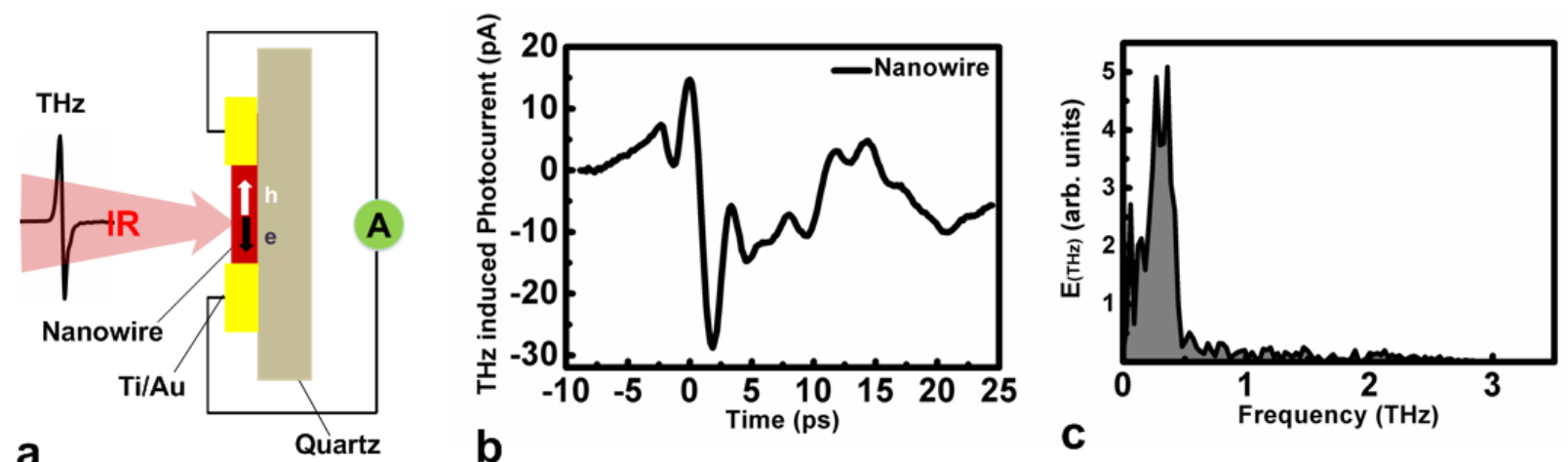

Fig. 2.a) A schematic diagram of a typical photoconductive single-nanowire detector in operation. b) THz induced current measured from a nanowire detector in the THz-TDS system. c) THz amplitude spectrum corresponding to b).

\section{Conclusion}

Single GaAs/AlGaAs-nanowire photoconductive $\mathrm{THz}$ detectors were successfully fabricated and incorporated in to the THz-TDS system. FDTD simulations were performed to provide insight into detector performances for optimal detection.

\section{References}

[1] X. L. Shen, et al., "Detection and Segmentation of Concealed Objects in Terahertz Images," IEEE T IMAGE PROCESS, 17, 2465-2475 (2008)

[2] P. Parkinson, et al., "Transient terahertz conductivity of GaAs nanowires," NANO LETT, 7, 2162-2165 (2007).

[3] D. H. Auston, "Picosecond optoelectronic switching and gating in silicon," APPL PHYS LETT, 26, 101-103 (1975)

[4] N. Jiang, et al., "Enhanced Minority Carrier Lifetimes in GaAs/AlGaAs Core-Shell Nanowires through Shell Growth Optimization," NANO LETT, 13, 5135-5140 (2013)

[5] J. Cunningham, et al., "On-chip terahertz systems for spectroscopy and imaging," ELECTRON LETT, 46, S34-S37 (2010)

[6] H. J. Joyce, et al., "Twin-free uniform epitaxial GaAs nanowires grown by a two-temperature process," NANO LETT, 7, 921-926 (2007).

[7] P. Parkinson, et al., "Direct-write non-linear photolithography for semiconductor nanowire characterization," NANOTECHNOLOGY, 23, 335704 (2012).

[8] K. Peng, et al., "Single nanowire photoconductive terahertz detectors," NANO LETT, just accepted (2014). 